\title{
Application of the Triple Collocation Method to Evaluate Accuracy of Altimeter, Buoy and Modeled Height of Wind Waves
}

\author{
Fedor A. Pogarskiy ${ }^{1}$, Vladislav G. Polnikov ${ }^{1}$, Arseniy A. Kubryakov ${ }^{2}$ \\ ${ }^{1}$ Obukhov Institute of Atmospheric Physics, Russian Academy of Sciences, Moscow, Russia \\ pogarskiy@ifaran.ru \\ polnikov@mail.ru \\ ${ }^{2}$ Marine Hydrophysical Institute, Russian Academy of Sciences, Sebastopol, Russia \\ arskubr@yandex.ru
}

\begin{abstract}
For independent and simultaneous assessment accuracy for the altimeter and buoys measurement data and the numerical simulation of the wind-wave heights, the triple collocation (TC) method described in paper [1] was applied. We used data from 6 missions of satellites, 74 buoys, reanalysis of ERA5 and the results of simulations using the numerical WaveWatch and WAM models in the northern parts of the Atlantic and Pacific Oceans. The stability of the TC method for estimating the root-mean-square errors (RMS) for wave heights is shown. It has been established that the reanalysis has the highest accuracy, the buoy data and simulation results are much lower, and the satellite data do even less. The reasons for the established accuracy distribution are analyzed.
\end{abstract}

Keywords: wind waves, buoy measurements, satellite measurements, numerical simulation, triple collocation, standard deviation

Copyright (C2019 by the paper's authors. Copying permitted for private and academic purposes 


\title{
ПРИМЕНЕНИЕ МЕТОДА ТРОЙНОЙ КОЛЛОКАЦИИ ДЛЯ ОЦЕНКИ ТОЧНОСТИ ДАННЫХ АЛЬТИМЕТРОВ, БУЕВ И МОДЕЛИРОВАНИЯ ВЫСОТЫ ВЕТРОВЫХ ВОЛН
}

\author{
Ф.А. Погарский', В.Г. Полников ${ }^{1}$, А.А. Кубряков ${ }^{2}$ \\ ${ }^{1}$ Институт физика атмосферы им.А.М. Обухова РАН, Москва, Россия \\ pogarskiy@ifaran.ru \\ polnikov@mail.ru \\ ${ }^{2}$ Морской гидрофизический институт РАН, Севастополь, Россия \\ arskubr@yandex.ru
}

\begin{abstract}
Для независимой и одновременной оценки точности альтиметрических и буйковых данных измерения и численного расчета высот ветровых волн применён метод тройной коллокации по работе [1]. Использованы данные 6 миссий спутников, 74 буев, реанализа ERA5 и результаты расчётов по численным моделям WaveWatch и WAM в северных частях Атлантического и Тихого океанов. Показана стабильность метода ТК для оценки среднеквадратичных ошибок (СКО) высот волн. Установлено, что наивысшую точность имеет реанализ, значительно меньшую - буйковые данные и результаты моделирования, и ещё меньшую - спутниковые данные. Анализируются причины установленного распределения точности.
\end{abstract}

Ключевые слова: ветровые волны, тройная коллокация, буйковые измерения, спутниковые измерения, численное моделирование, среднеквадратичное отклонение

\section{Введение}

Решение проблемы оценки точности данных измерений и численного моделирования высот ветрового волнения на глобальных масштабах имеет большое практическое значение для безопасности мореплавания и промышленной морской деятельности. При этом даже контактные (буйковые) измерения в открытом океане не всегда могут считаться достоверными, в силу подвижности измерительного прибора. Поэтому нужны такие методы контроля точности, которые используют сопоставление данных различной природы их получения. Одним из таких методов является тройная коллокация (сопоставление) данных различного рода, описанная в [1]. Целью данной работы является применение метода тройной коллокации (ТК) для получения одновременной оценки точности данных измерений значительных высот $H_{\mathrm{S}}$ ветровых волн для спутниковых альтиметров, океанских буев и результатов численного моделирования.

\section{Используемые данные и методика работы}

В работе использованы следующие данные о значительных высотах $H_{\mathrm{S}}$ ветровых волн:

1) альтиметрические спутниковые измерения второго уровня обработки (А-данные) шести миссий (Altika, Envisat, GFO, H2Y, Jason-1, Jason-2) [2];

2) контактные измерения на волнографических буях (В-данные) из базы NDBC [3] (рис. 1);

3) результаты численного моделирования (М-данные) трёх видов:

3a) реанализ ERA5 разрешением $0,1^{\circ}$ по пространству и 1 ч по времени [4]; 
3б) результаты оперативных расчётов по модели WaveWatch3 (WW) с прогностическим ветром, полученном по модели GFS разрешением 10' и 3 ч соответственно [5];

3в) результаты расчётов по ранее модифицированной авторами версии модели WAM (newWAM) [6] с ветром из реанализа ERA5 разрешением 0,1 и 1-3 ч соответственно.

Все данные взяты за период 2012-2016 годов.

В качестве метода исследования использована техника тройной коллокации (ТК) по работе [1]. Полагается, что каждое значение всех типов данных является линейной функцией от истинного значения вида:

$$
A_{i}=\beta_{A} T_{i}+e_{A_{i}} ; \quad B_{i}=\beta_{B} T_{i}+e_{B_{i}} ; \quad M_{i}=\beta_{M} T_{i}+e_{M_{i}},
$$

где $A, B$ и $M$ - значения $H_{\mathrm{S}}$ соответствующего типа; $T$ - истинное значение $H_{\mathrm{S}}, \beta$ - калибровочная константа, $e$ - случайная ошибка измерения (расчёта). Предполагается также, что А-, В- и Мданные имеют, в среднем, некоррелированные ошибки:

$$
\left\langle e_{B_{i}} e_{A_{i}}\right\rangle=\left\langle e_{B_{i}} e_{M_{i}}\right\rangle=\left\langle e_{A_{i}} e_{M_{i}}\right\rangle=0,
$$

где осреднение проводится по ансамблю значений всех видов данных, перекрывающихся по пространству-времени. Для этой цели была выполнена процедура формирования рядов для такого рода перекрывающихся данных (коллокация).

Затем выполняется следующая итерационная процедура. Один тип данных (например, буйковые, В-данные) принимается за эталонный (т.е. $\beta_{\mathrm{B}}=1$ ), и рассчитываются калибровочные константы $\beta_{\mathrm{A}}$ и $\beta_{\mathrm{M}}$, минимизирующие ошибки всех трёх типов данных. В таком случае, например, калибровочная константа $\beta_{\mathrm{A}}$ является корнем квадратного уравнения $a \beta_{\mathrm{A}}{ }^{2}+b \beta_{\mathrm{A}}+c=0$, где

$$
\begin{aligned}
& a=\frac{\left\langle\left(B_{i}-A_{i}\right)\left(B_{i}-M_{i}\right)\right\rangle}{\left\langle\left(A_{i}-B_{i}\right)\left(A_{i}-M_{i}\right)\right\rangle}\left\langle B_{i} A_{i}\right\rangle \\
& b=\left\langle B_{i}^{2}\right\rangle-\frac{\left\langle\left(B_{i}-A_{i}\right)\left(B_{i}-M_{i}\right)\right\rangle}{\left\langle\left(A_{i}-B_{i}\right)\left(A_{i}-M_{i}\right)\right\rangle}\left\langle A_{i}^{2}\right\rangle, \\
& c=\left\langle B_{i} A_{i}\right\rangle
\end{aligned}
$$

а угловые скобки означают осреднение по всему массиву используемых данных. Аналогично рассчитывается калибровочная константа $\beta_{\mathrm{M}}$.

Далее, А- и М-данные нормируются на полученные калибровочные константы, и процедура итерации повторяется, пока процесс не сойдётся (т.е, новые калибровочные константы не станут равными 1). Искомые значения среднеквадратичных ошибок (СКО) В-, А- и М-данных, соответственно, имеют вид

$$
E_{B}=\sqrt{\left\langle\left(B_{i}-A_{i}\right)\left(B_{i}-M_{i}\right)\right\rangle} ; E_{A}=\sqrt{\left\langle\left(A_{i}-B_{i}\right)\left(A_{i}-M_{i}\right)\right\rangle} ; E_{M}=\sqrt{\left\langle\left(M_{i}-B_{i}\right)\left(M_{i}-A_{i}\right)\right\rangle} .(4)
$$

Здесь значения $A_{\mathrm{i}}$ и $M_{\mathrm{i}}$ взяты с учётом рассчитанных калибровочных констант.

Отметим, что возможна только относительная калибровка. Тем не менее, рассчитанные ошибки практически не зависят от типа данных, выбранных эталонными. Например, при выборе эталонными А-данные вместо В-данных, ошибки всех трёх типов данных остаются прежними, и калибровочная константа В-данных оказывается обратной калибровочной константе А-данных в случае, когда В-данные были выбраны в качестве эталона.

Проведены три аналогичных расчёта отдельно для каждого вида М-данных. По техническим причинам временной шаг М-данных (см. таблицу) и набор используемых буёв для разных видов М-данных (см. рис. 1) оказались различными. Поэтому для корректного их сравнения дополнительно проведена процедура коллокации только по совпадающему для всех видов набору буйковых и соответствующих им спутниковых измерений. 


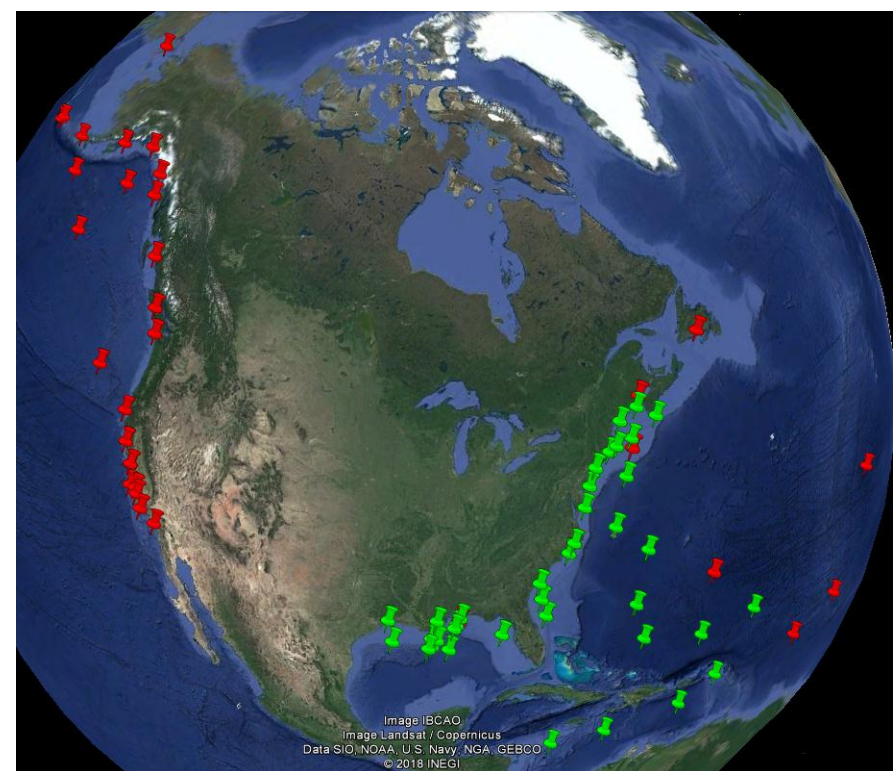

Рис. 1. Расположение буёв из базы NDBC. Зелёным цветом указаны буи, использованные при коллокации со всеми типами М-данных, красным - с некоторыми типами М-данных.

Таблица. Массивы данных измерений, использованные для расчётов СКО с разными видами М-данных

\begin{tabular}{|l|c|c|c|c|}
\hline Вид М-данных & $\begin{array}{c}\text { Реанализ } \\
\text { ERA5 } \\
\text { (шаг 1 ч) }\end{array}$ & $\begin{array}{c}\text { Модель } \\
\text { WaveWatch } \\
\text { (шаг 3 ч) }\end{array}$ & $\begin{array}{c}\text { Модель } \\
\text { nеwWAM } \\
\text { (шаг 1-3 ч) }\end{array}$ & $\begin{array}{c}\text { Все виды по } \\
\text { совпадающему } \\
\text { набору измерений }\end{array}$ \\
\hline Количество буёв & 75 & 61 & 47 & 38 \\
\hline $\begin{array}{l}\text { Количество миссий } \\
\text { спутниковых } \\
\text { измерений }\end{array}$ & \multicolumn{5}{|l}{} \\
\hline $\begin{array}{l}\text { Общее количество } \\
\text { данных }\end{array}$ & 11700 & 3618 & 6150 & 2636 \\
\hline
\end{tabular}

\section{Результаты и обсуждение}

На рисунке 2 представлены результаты расчётов СКО для значительной высоты волн $H_{\mathrm{S}}$; a по всему набору данных, б - только по совпадающему (для трёх видов М-данных) набору буйковых и спутниковых измерений (см. таблицу).

Результаты показывают стабильность метода ТК для оценки СКО (см. рис. 2б). При серьёзном изменении характера М-данных (оценки $E_{\mathrm{M}}$ СКО для моделей превышают таковую для реанализа более чем в 2 раза), рассчитанные ошибки буйковых $E_{\mathrm{B}}$ и альтиметрических $E_{\mathrm{A}}$ измерений сохраняются практически неизменными (разность не превышает 8\%). В то же время, хорошо заметна чувствительность к изменению набора буйковых и альтиметрических данных (см. динамику величин $E_{\mathrm{B}}$ и $E_{\mathrm{A}}$ на рис. 2 а и в сравнении с рис. 26 ).

Относительно невысокая точность буйковых измерений $\left(E_{\mathrm{B}}=0,26-0,34\right.$ м), тем не менее, часто принимаемых за точные (!), как мы полагаем, связана с их подвижностью и техническими ограничениями восстановления значений малых высот волн [3].

Не очень высокая точность численного моделирования ( $E_{\mathrm{M}}=0,29-0,31$ м), по-видимому, определяется сочетанием двух факторов. С одной стороны, наличие высокого пространственного разрешения и хорошее качество современных численных моделей [6]. С другой стороны, краевые эффекты расположения значительного количества используемых буёв вблизи (2-3 шага модельной сетки) береговой линии, ухудшающие качество результатов моделирования в этих местах. При этом ожидаемо высокая точность данных реанализа $\left(E_{\mathrm{M}}=0,11-0,15 \mathrm{~m}\right)$, очевидно, связана с уменьшением случайных ошибок вследствие усвоения различных данных наблюдений, принятом в составлении реанализа [4]. 

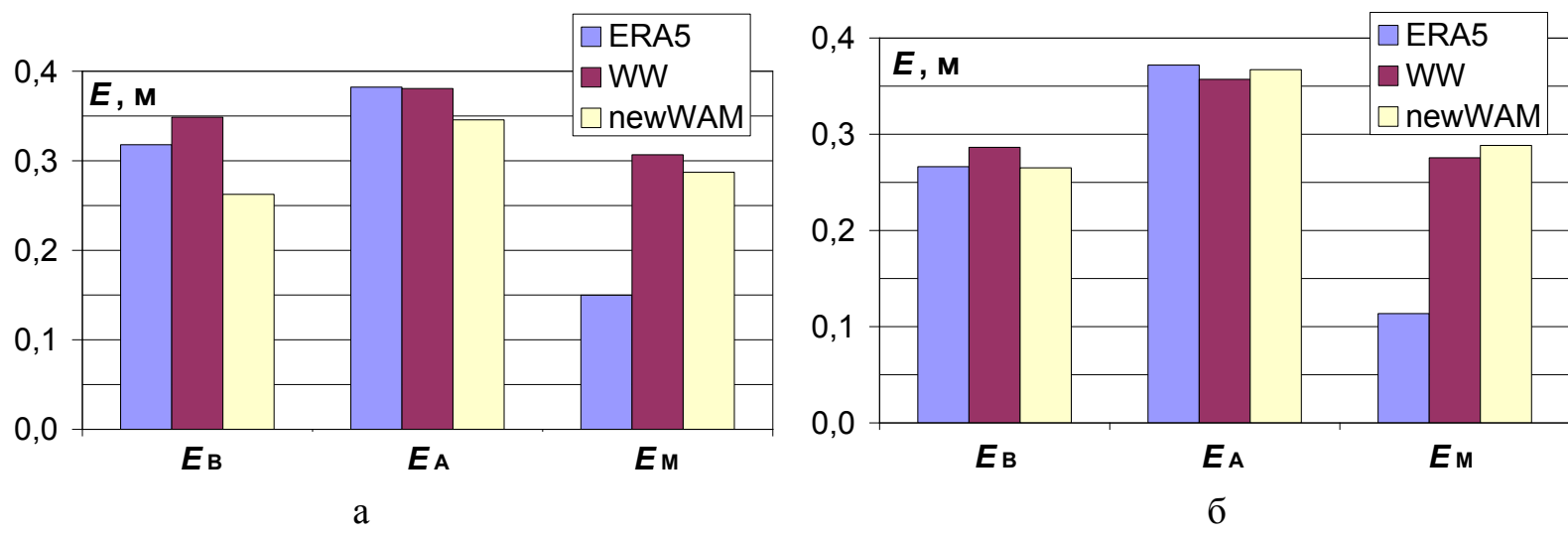

Рис. 2. Среднеквадратические ошибки буйковых измерений $\left(E_{\mathrm{B}}\right)$, спутниковых измерений $\left(E_{\mathrm{A}}\right)$ и численного моделирования $\left(E_{\mathrm{M}}\right)$ для значительной высоты волн $H_{\mathrm{S}}$; а - по всему набору данных, $\sigma-$ только по совпадающему набору буйковых и спутниковых измерений.

Цветом показаны результаты расчётов с разными видами М-данных

Неожиданно низкая точность альтиметров $\left(E_{\mathrm{A}}=0,35-0,38\right.$ м) (по сравнению с работой [1]) обусловлена, вероятно, объединением данных шести миссий, имеющих разные калибровки. Кроме того, спутниковые калибровки измерений высот волн могут иметь сложную зависимость от интенсивности волнения, имеющую сдвиг и неодинаковый наклон при различных высотах волн [7]. Учёт этих особенностей авторы планируют при проведении дальнейших исследований.

Работа выполнена при поддержке проекта РФФИ №18-05-00161 и базового бюджетного финансирования НИОКТР гос. регистрация № АААА-А18-118021290158-2.

\section{References}

[1] Janssen P. A. E. M., Abdalla S, Hersbach H, and Bidlot J.-R. Error Estimation of Buoy, Satellite, and Model Wave Height Data, J. Atmosph. Oceanic Thechnol. 2007, V. 24(9), pp. 1665-1677. DOI: 10.1175/JTECH2069.1

[2] http://globwave.ifremer.fr/

[3] https://www.ndbc.noaa.gov/

[4] Copernicus Climate Change Service (C3S) (2017): ERA5: Fifth generation of ECMWF atmospheric reanalyzes of the global climate. Copernicus Climate Change Service Climate Data Store (CDS). https://cds.climate.copernicus.eu/cdsapp\#!/home

[5] ftp://polar.ncep.noaa.gov/pub/history/waves/

[6] Samiksha, S. V., Polnikov V., Pogarskii F., et al. Verification of model wave heights with long-term moored buoy data: application to wave field over the Indian Ocean, Ocean Engineering, 2015,V. 104, pp. 469-479. http://dx.doi.org/10.1016/j.oceaneng.2015.05.020.

[7] Polnikov V.G., Pogarskiy F. A., Zilitinkevich N. S., Kubryakov A.A. Using the Along-Track Altimeter Data for a Verification of the Numerical Wave Models, Issledovanie Zemli iz Kosmosa, 2018, No. 4, pp. 21-318 (in Russian). DOI:10.31857/S020596140002353-9. 